\title{
The Impact of Seropositivity on Bone Mineral Density in Rheumatoid Arthritis - A 3-year Prospective Observational Study
}

\author{
Shan-Fu Yu \\ Kaohsiung Chang Gung Memorial Hospital \\ Jia-Feng Chen \\ Kaohsiung Chang Gung Memorial Hospital \\ Ying-Chou Chen \\ Kaohsiung Chang Gung Memorial Hospital \\ Yu-Wei Wang \\ Kaohsiung Chang Gung Memorial Hospital \\ Chung-Yuan Hsu \\ Kaohsiung Chang Gung Memorial Hospital \\ Han-Ming Lai \\ Kaohsiung Chang Gung Memorial Hospital \\ Hsiao-Ru He \\ Kaohsiung Chang Gung Memorial Hospital \\ Tien-Tsai Cheng ( $\square$ tiantsai0919@gmail.com ) \\ Kaohsiung Chang Gung Memorial Hospital
}

\section{Research Article}

Keywords: rheumatoid arthritis, bone mineral density, anti-cyclic citrullinated peptide antibodies, rheumatoid factor, fracture

Posted Date: November 1st, 2021

DOI: https://doi.org/10.21203/rs.3.rs-944340/v1

License: (c) (i) This work is licensed under a Creative Commons Attribution 4.0 International License. Read Full License 


\section{Abstract}

\section{Background:}

To explore the impact of seropositivity on systemic bone loss in rheumatoid arthritis (RA).

\section{Methods:}

This was an interim analysis of the RA registry. Clinical characteristics in the registry were documented, and bone mineral density (BMD) was measured; this was repeated 3 years later. Participants were grouped into seropositive (SPRA) and seronegative (SNRA) based on the presence or absence of rheumatoid factor (RF) and/or anti-cyclic citrullinated peptide antibodies (ACPA). After matching (1:2) for age and sex, SNRA and SPRA were regrouped into $A$ and $B$. To elucidate the impact of number of antibodies on BMD changes, the matched group was sub-divided according to the number of antibodies present ( 0 , group I; 1 , group II; 2 , group III). The changes in BMD were compared for each group at baseline and 3 years later.

\section{Results:}

A total of 477 participants who completed a 3-year observation period were included. After matching, 312 participants were enrolled (group A, 104 ; group B, 208). Three years later, group B had significant bone loss in the femoral neck (FN) $(p<0.001)$, total hip (TH) $(p=0.001)$, and 1st4th lumbar vertebrae (L1-4) $(p=0.006)$, while group A has bone loss only at FN $(p=0.002)$. Groups I, II, and III included 104, 52 , and 156 participants, respectively. Significant bone loss was recorded at FN $(p=0.002)$ in group I, FN $(p<0.001)$ in group II, and FN $(p<0.001)$, TH ( $p=$ $0.002)$, and L1-4 ( $p=0.016)$ in group III. In terms of percent change in BMD $(\triangle \mathrm{BMD} \%)$, more significantly negative changes were found at $\mathrm{TH}$ in group $\mathrm{B}(p=0.027)$ and within groups $\mathrm{I}-\mathrm{III}(p$ for trend $=0.021)$. Logistic regression showed that seropositivity is a significant predictor of $\triangle \mathrm{BMD} \geqq-5 \%$ at $\mathrm{TH}$ (odds ratio $1.85,95 \%$ confidence interval $1.03-3.33, p=0.039$ ).

\section{Conclusions:}

SPRA lost more bone than SNRA after 3 years. More attention should be paid to SPRA patients, especially those with double-positive antibodies, with vigorous evaluation of BMD and fracture risk.

\section{Introduction}

Rheumatoid arthritis (RA) is a chronic systemic disease that can lead to local bone erosion and generalized osteoporosis. Rheumatoid factor (RF) and anti-citrullinated protein antibodies (ACPA) are the two most notable autoantibody systems commonly used as aids for diagnosing or classifying RA as well as for providing a variety of clinical and pathophysiological information [1]. Patients positive for ACPA and/or RF may be labeled together as "seropositive" RA (SPRA) and compose approximately $50 \%$ to $80 \%$ of the RA population [1]. Several studies have indicated that SPRA has a greater severity in terms of disease activity and function impairment, as well as increased mortality over time [2, 3] and poor response to treatment compared to seronegative RA (SNRA) [4]. Although both SPRA and SNRA fulfill the 1987 American College of Rheumatology (ACR) revised classification criteria [5] or the 2010 ACR/European League Against Rheumatism (EULAR) classification criteria [6], it seems that their clinical manifestations, courses, and prognostic features are distinctive.

It has been reported that the annual rate of bone loss in patients with active RA ranges between 5.5\% and 10\% [7]. Meanwhile, RF and/or ACPA are associated with juxta-articular osteoporosis, erosions, and generalized bone loss [8, 9]. Several studies have shown that ACPAs and RF synergize to promote RA-associated inflammation, disease activity, and clinical onset [10,11] and can be associated with bone erosion and structural damage of RA [12]. The combination of ACPA and RF could predict the therapeutic responses to rituximab and abatacept [13]. However, the clinical predictive value of each of the antibodies (singly or in combination) on systemic bone loss has not been well studied.

In this study, we aimed to explore long-term bone mineral density (BMD) changes in patients with SPRA and SNRA, and investigate the association between the number of antibodies and systemic bone loss in patients with RA.

\section{Materials And Methods Study population and design}

The inclusion criteria for participants and the methods used in this study were the same as those reported previously [14]. Study participants enrolled in a RA registry at Kaohsiung Chang Gung Memorial Hospital starting from 1 September 2014 . A total of 651 patients who satisfied the 1987 revised ACR criteria for RA [5] or the 2010 ACR/EULAR classification criteria for RA [6], were enrolled in this study. Relevant information was recorded, including demographic characteristics such as age, sex, comorbidities, and body mass index, as well as disease-specific 
information such as the age at diagnosis, disease duration, disease activity measured by erythrocyte sedimentation rate (ESR), C-reactive protein, and the disease activity score-28 joint-ESR (DAS28-ESR), medications including glucocorticoids (GC) and biological and targeted synthetic disease-modifying antirheumatic drugs (b/tsDMARDs), and presence/absence of ACPA and RF. Lifestyle, evidence of previous fragility fracture (history or radiographic), and risk factors of fragility fracture on the Fracture Risk Assessment Tool (FRAXß) were also recorded. The 10-year probabilities of major and hip fractures in each patient, calculated using the FRAXß with BMD (Taiwan version), were collected. Seropositivity was defined as any value $>15 \mathrm{IU} / \mathrm{mL}$ for RF and $>7 \mathrm{IU} / \mathrm{mL}$ for ACPA.

The BMD of each patient was measured at enrolment and after the 3-year follow-up using a dual-energy X-ray absorptiometry scanner (Delphi A; Hologic Corp., Waltham, MA, USA) for femoral neck (FN), total hip (TH), and 1st - 4th lumbar vertebrae (L1-4). For postmenopausal women and men aged 50 years and older, osteoporosis was defined as a T-score of -2.5 , or less at the FN based on the normal reference database for young white females $[15,16]$. We calculated the percentage change in BMD (\% $\triangle \mathrm{BMD})$ for each participant as follows: [(second BMD baseline BMD) / baseline BMD] × 100 between assessments. In this study, we defined that $\triangle \mathrm{BMD} \geqq-5 \%$ at three sites as excessive bone loss, based upon the previous studies regarding smallest detectable difference of BMD measurement $[17,18]$. We defined a new incident fracture as any symptomatic non-traumatic fracture, including the forearm, hip, pelvic, and humerus fractures or asymptomatic morphometric vertebral fracture. A morphometric fracture was assessed on lateral radiographs of the lumbar spine according to Genant's semiquantitative assessment of vertebral fractures [19]. An independent radiologist assessed the evidence of morphometric vertebral compression fractures at enrolment and subsequently on an as-needed basis, with follow-up spinal radiographs during the 3-year observation period and at the end of the study. The local Institutional Review Board of Chang Gung Memorial Hospital approved the study (104-3530B, 201901054B0), which was performed according to the principles of the Declaration of Helsinki. Informed consent was obtained from all participants.

Patients who were positive for RF or ACPA were grouped into the SPRA group, while those who were negative were grouped as SNRA. The participants were sub-grouped into $A$ and $B$ after matching for age and sex. Finally, the matched group was sub-grouped according to the number of antibodies present (0, group I; 1 , group II; 2 , group III).

\section{Statistics}

The data were checked for normality, which showed that the baseline characteristics had a skewed distribution; therefore, they were analyzed using non-parametric methods. Independent two-sample t-test and one-way analysis of variance (ANOVA) test were used to compare continuous variables with normal distribution and expressed as mean \pm standard deviation (SD). The Mann-Whitney U test and Kruskal-Wallis test were used to compare continuous variables with skewed distribution; they were expressed as median (interquartile range, IQR). The chisquare or Fisher's exact test was used to assess the association between categorical variables. The intra-and inter-group BMD changes from enrollment to 3 years later were compared with a linear mixed model. Trend analyses were performed using ANOVA for each category of number of antibodies. A binary logistic regression analysis (enter model) was performed using excessive bone loss as the dependent variable. Ordinal regression was performed to compare baseline characteristics between patients harbouring 0-2 autoantibodies and to yield odds ratio (OR) and 95\% confidence interval (CI), which signify the increase in association with every additional autoantibody. Data that resulted in $p<0.10$ in univariate analysis were studied through logistic regression to access their independence. All statistical analyses were performed using IBM SPSS version 22 software (IBM Co., Armonk, NY, USA). A p-value of < 0.05, was considered significant in all analyses.

\section{Results}

\section{Demographics and clinical characteristics of SPRA and SNRA participants}

The participants' disposition is shown in Fig. 1. A total of 651 participants were registered for the study cohort, which would last until the end of April 2020, and a total of 477 participants completed the 3-year observation period. To compare BMD changes in SNRA and SPRA, taking the mutual interference of age and sex into account, we controlled for these two factors. We obtained 312 matched participants, of whom 104 were allocated to group A and 208 to group B (Fig. 1). The baseline DAS28-ESR and ESR were significantly higher in group B than in group A ( $p=$ 0.002 and $p=0.001$, respectively) (Table 1 ). The proportion of patients using $\mathrm{b} /$ tsDMARDs in group $\mathrm{B}$ was higher than that in group $\mathrm{A}(p=$ 0.007). No significant difference was found between the two groups in terms of history of previous fractures, use of anti-osteoporotic therapy, and baseline GC (Table 1).

Table 1

clinical characteristics of participants before and after matching 


\begin{tabular}{|c|c|c|c|c|c|c|c|c|}
\hline & \multicolumn{4}{|c|}{ Before matching } & \multicolumn{4}{|l|}{ After matching } \\
\hline & $\begin{array}{l}\text { Total } \\
(n=469)\end{array}$ & $\begin{array}{l}\text { SNRA } \\
(n=110)\end{array}$ & $\begin{array}{l}\text { SPRA } \\
(n=359)\end{array}$ & $p^{\mathrm{a}}$ & $\begin{array}{l}\text { Total } \\
(n=312)\end{array}$ & $\begin{array}{l}\text { Group A } \\
(n=104)\end{array}$ & $\begin{array}{l}\text { Group B } \\
(n=208)\end{array}$ & $p^{\mathrm{a}}$ \\
\hline Age, years & $58(14)$ & $55(14)$ & $60(13)$ & 0.014 & $57(14)$ & $55(14)$ & $57(14)$ & 0.504 \\
\hline Female, n (\%) & $398(84.9)$ & $96(87.3)$ & 302 (84.1) & 0.420 & $275(88.1)$ & $90(86.5)$ & 185 (88.9) & 0.536 \\
\hline $\begin{array}{l}\text { Postmenopause, } \\
\mathrm{n}(\%)\end{array}$ & $313(78.6)$ & $67(69.8)$ & $246(81.5)$ & 0.015 & 208 (75.6) & 64 (71.1) & $164(77.8)$ & 0.223 \\
\hline $\mathrm{BMI}, \mathrm{kg} / \mathrm{m}^{2}$ & $23.1(5.1)$ & $23.7(5.0)$ & $23(5.1)$ & 0.155 & $23.4(5.2)$ & $23.8(5.2)$ & $23.0(5.4)$ & 0.123 \\
\hline $\begin{array}{l}\text { Comorbidities +, } \\
\text { n (\%) }\end{array}$ & 278 (59.3) & $63(57.3)$ & 215 (59.9) & 0.625 & 190 (60.9) & $63(60.6)$ & 127 (61.1) & 0.935 \\
\hline \multicolumn{9}{|l|}{$\begin{array}{l}\text { RA- related } \\
\text { factors }\end{array}$} \\
\hline $\begin{array}{l}\text { Disease duration, } \\
\text { years }\end{array}$ & $13(3)$ & $12(13)$ & $13(13)$ & 0.870 & $12(11)$ & $12(13)$ & $12(12)$ & 0.765 \\
\hline $\begin{array}{l}\text { Baseline DAS28- } \\
\text { ESR }\end{array}$ & $3.2(1.7)$ & $2.7(1.6)$ & $3.3(1.6)$ & $<0.001$ & $3.0(1.6)$ & $2.7(1.7)$ & $3.3(1.5)$ & 0.002 \\
\hline $\begin{array}{l}\text { Mean DAS28- } \\
\text { ESR }\end{array}$ & $3.0(1.2)$ & $2.8(1.2)$ & $3.0(1.2)$ & 0.017 & $2.9(1.2)$ & $2.9(1.2)$ & $3.0(1.1)$ & 0.088 \\
\hline HAQ-DI & $0.25(0.88)$ & $0.13(0.75)$ & $0.25(1.0)$ & 0.092 & $0.25(0.88)$ & $0.13(0.66)$ & $0.25(0.88)$ & 0.295 \\
\hline $\mathrm{ESR}, \mathrm{mm} / \mathrm{h}$ & $17(21)$ & $11.0(13.0)$ & $18.0(24.0)$ & $<0.001$ & $16(19)$ & $11(13.8)$ & $17(20.5)$ & 0.001 \\
\hline \multicolumn{9}{|l|}{$\begin{array}{l}\text { Fracture-related } \\
\text { factors }^{\mathrm{b}}+\end{array}$} \\
\hline $\begin{array}{l}\text { Parent fractured } \\
\text { hip + }\end{array}$ & $36(7.7)$ & $10(9.2)$ & $26(7.3)$ & 0.522 & $26(8.3)$ & $10(9.7)$ & $16(7.8)$ & 0.571 \\
\hline $\begin{array}{l}\text { Previous fracture } \\
+\end{array}$ & 148 (31.6) & 36 (32.7) & $112(31.2)$ & 0.763 & $100(32.1)$ & 34 (32.7) & 66 (31.7) & 0.864 \\
\hline Alcohol + & $7(1.5)$ & $2(1.8)$ & $5(1.4)$ & 0.747 & $4(1.3)$ & $2(1.9)$ & $2(1.0)$ & 0.477 \\
\hline Smoking + & $31(6.6)$ & $4(3.6)$ & $27(7.5)$ & 0.190 & $15(4.8)$ & $4(3.8)$ & $11(5.3)$ & 0.575 \\
\hline $\begin{array}{l}\text { Secondary } \\
\text { osteoporosis + }\end{array}$ & $21(4.5)$ & $3(2.7)$ & $18(5.0)$ & 0.432 & $8(2.6)$ & $2(1.9)$ & $6(2.9)$ & 0.612 \\
\hline \multicolumn{9}{|l|}{ BMD (g/m2) } \\
\hline $\mathrm{FN}$ & $0.633 \pm 0.120$ & $0.648 \pm 0.122$ & $0.620 \pm 0.117$ & 0.014 & $0.641 \pm 0.123$ & $0.664 \pm 0.114$ & $0.627 \pm 0.125$ & 0.004 \\
\hline TH & $0.789 \pm 0.141$ & $0.801 \pm 0.140$ & $0.775 \pm 0.142$ & 0.067 & $0.796 \pm 0.143$ & $0.818 \pm 0.128$ & $0.784 \pm 0.147$ & 0.015 \\
\hline L1-4 & $0.871 \pm 0.170$ & $0.885 \pm 0.167$ & $0.860 \pm 0.168$ & 0.129 & $0.874 \pm 0.868$ & $0.894 \pm 0.167$ & $0.863 \pm 0.163$ & 0.104 \\
\hline $\begin{array}{l}\text { Osteoporosis }{ }^{\mathrm{c}}, \mathrm{n} \\
(\%)\end{array}$ & $138(29.4)$ & $22(16.2)$ & $116(24.9)$ & 0.033 & $81(26)$ & $16(15.4)$ & 65 (31.3) & 0.003 \\
\hline \multicolumn{9}{|l|}{ FRAX score } \\
\hline Major & $14(18.9)$ & $12(13.5)$ & $15(19)$ & 0.007 & $13(18.1)$ & $12(13.9)$ & $14(20.2)$ & 0.086 \\
\hline Hip & $4.2(8.4)$ & $3.4(6.2)$ & $5.0(9.5)$ & 0.001 & $3.9(8)$ & $3.4(6.1)$ & $4.3(9.5)$ & 0.018 \\
\hline $\begin{array}{l}\text { New incident } \\
\text { fracture (3 } \\
\text { years), } \mathrm{n}(\%)\end{array}$ & $132(28.1)$ & $24(26.1)$ & 108 (34.8) & 0.116 & 86 (27.6) & $22(25.3)$ & $64(36)$ & 0.082 \\
\hline \multicolumn{9}{|l|}{ Medications } \\
\hline GC +, n (\%) & $409(87.2)$ & $90(81.8)$ & 319 (88.9) & 0.053 & $291(93.3)$ & $92(88.5)$ & 199 (95.7) & 0.017 \\
\hline $\begin{array}{l}\mathrm{b} / \text { tsDMARDs }^{\mathrm{d}_{1}}+ \\
\mathrm{n}(\%)\end{array}$ & 84 (17.9) & $12(10.9)$ & $72(20.1)$ & 0.031 & 79 (25.3) & $16(15.4)$ & $63(30.3)$ & 0.004 \\
\hline
\end{tabular}




\begin{tabular}{|c|c|c|c|c|}
\hline & Group I & Group II & Group III & \\
\hline & $(n=104)$ & $(n=52)$ & $(n=156)$ & $p^{\mathrm{a}}$ \\
\hline Age, years & $55(14)$ & $58(13)$ & $57(14)$ & 0.740 \\
\hline Female, n (\%) & $90(86.5)$ & $50(96.2)$ & $135(86.5)$ & 0.147 \\
\hline Postmenopause, $n(\%)$ & $63(71.1)$ & $39(78)$ & $105(77.8)$ & 0.475 \\
\hline $\mathrm{BMI}, \mathrm{kg} / \mathrm{m}^{2}$ & $23.8(5.2)$ & $22.8(5.3)$ & $23(5.4)$ & 0.297 \\
\hline Comorbidities +, n (\%) & $63(60.6)$ & $41(78.8)$ & $86(55.1)$ & 0.010 \\
\hline \multicolumn{5}{|l|}{ RA-related factors + } \\
\hline Disease duration, years & $12(13)$ & $11.5(19)$ & $12(10)$ & 0.874 \\
\hline Baseline DAS28-ESR & $2.7(1.7)$ & $3.0(1.6)$ & $3.3(1.6)$ & 0.006 \\
\hline Mean DAS28 at follow-up & $2.8(1.2)$ & $2.9(1.0)$ & $3.0(1.2)$ & 0.233 \\
\hline HAQ-DI & $0.13(0.66)$ & $0.25(1.03)$ & $0.25(0.88)$ & 0.442 \\
\hline $\mathrm{ESR}, \mathrm{mm} / \mathrm{h}$ & $11(13.8)$ & $14(20.3)$ & $18(22.8)$ & $<0.001$ \\
\hline \multicolumn{5}{|l|}{ Fracture-related factors ${ }^{b_{+}}$} \\
\hline Parent fractured hip & $10(9.7)$ & $2(3.8)$ & $14(9.2)$ & 0.420 \\
\hline Previous fracture & $34(32.7)$ & $16(30.8)$ & $50(32.1)$ & 0.971 \\
\hline Alcohol & $2(1.9)$ & $0(0)$ & $2(1.3)$ & 0.603 \\
\hline Smoking & $4(3.8)$ & $0(0)$ & $11(7.1)$ & 0.103 \\
\hline Secondary osteoporosis & $2(1.9)$ & $1(1.9)$ & $5(3.2)$ & 0.774 \\
\hline \multicolumn{5}{|l|}{ Baseline BMD (g/m2) } \\
\hline $\mathrm{FN}$ & $0.664 \pm 0.114$ & $0.635 \pm 0.118$ & $0.624 \pm 0.127$ & 0.010 \\
\hline $\mathrm{TH}$ & $0.818 \pm 0.128$ & $0.782 \pm 0.144$ & $0.784 \pm 0.148$ & 0.053 \\
\hline L1-4 & $0.894 \pm 0.167$ & $0.868 \pm 0.172$ & $0.862 \pm 0.160$ & 0.254 \\
\hline \multicolumn{5}{|l|}{ FRAX score } \\
\hline Major & $12(13.9)$ & $13(18.6)$ & $14(20.7)$ & 0.214 \\
\hline Hip & $3.4(6.1)$ & $4.4(9.0)$ & $4.3(9.3)$ & 0.049 \\
\hline New incident fracture (3 years), $\mathrm{n}(\%)$ & $22(25.3)$ & $14(31.1)$ & $50(37.6)$ & 0.159 \\
\hline Osteoporosis ${ }^{\mathrm{c}}+, \mathrm{n}(\%)$ & $16(15.4)$ & $16(30.8)$ & $49(31.4)$ & 0.011 \\
\hline \multicolumn{5}{|l|}{ Medications } \\
\hline $\mathrm{GC}+, \mathrm{n}(\%)$ & $92(88.5)$ & $49(94.2)$ & $150(96.2)$ & 0.050 \\
\hline b/tsDMARDs ${ }^{d}+, n(\%)$ & $10(9.6)$ & $11(21.2)$ & $35(22.4)$ & 0.025 \\
\hline $\mathrm{AOT}_{+}+\mathrm{n}(\%)$ & $42(40.4)$ & $25(48.1)$ & $67(42.9)$ & 0.658 \\
\hline
\end{tabular}

Values are presented as mean \pm standard deviation, and median ((interquartile range), unless otherwise mentioned.

Abbreviation: BMI, body mass index; DAS28-ESR, disease activity score-28 joint-erythrocyte sedimentation; HAQ-DI: health assessment questionnaire disability index; ESR, erythrocyte sedimentation rate; BMD, bone mineral density; FN, femoral neck; TH: total hip; L1-4, 1st-4th lumbar vertebra; FRAX, fracture risk assessment tool; GC, glucocorticoid; b/tsDMARDs, biologic/target synthetic disease modify antirheumatic drugs, AOT, Anti-osteoporotic therapy

+ , presence

${ }^{a}$, comparison among 3 groups; ${ }^{b}$, defined as in FRAX tool; ${ }^{c}$, T-score (femoral neck) $\leq-2.5$; ${ }^{d}$, including anti-TNFa (etanercept, adalimumab, golimumab, certolizumab), anti-IL6 receptor (tocilizumab), CTLA4 analogue (abatacept), anti-CD 20 (rituximab) and JAK inhibitor (tofacitinib); ${ }^{e}$, including bisphosphonates, denosumab, teriparatide, during observation period 


\section{Changes of BMD in groups $A$ and $B$}

Group B had a significantly higher proportion of patients with osteoporosis $(p=0.003)$ and a higher 10-year probability of hip fracture $(p=$ 0.018), but had lower BMD at FN and TH $(p=0.004, p=0.005)$ at enrollment, compared to group A (Table 1 , right column). Three years later, the $\mathrm{BMD}$ at FN, TH, and L1-4 was significantly decreased from baseline in group $\mathrm{B}(p<0.001, p=0.001, p=0.006)$, respectively (Fig. 2a); a significant decrease was found only in FN in group A ( $p=0.002)$ (Fig. 2a). Intergroup differences over 3 years were significant at FN and TH ( $p=0.003$ and $p=0.005$, respectively), but were insignificant at L1-4 ( $p=0.105$ ) (Fig. 2a). In terms of $\triangle \mathrm{BMD} \%$ after 3 years, group B demonstrated significant bone loss at TH compared to group A ( $p=0.027$ ) (Fig. 3a), while there was no obvious $\triangle \mathrm{BMD} \%$ between groups $\mathrm{A}$ and $B$ at $F N$ and $L 1-4$. The difference in the rate of new incident fractures was not obvious between groups $A$ and $B$ over time (Table 1 , right column).

\section{Changes of BMD in groups I, II, and III}

After 3 years, compared with the baseline, significant BMD reductions were seen in group III participants at FN, TH, and L1 -4 ( $p<0.001, p=$ $0.002, p=0.016$, respectively). However, it was demonstrated that groups I and II had significant BMD reductions only at FN $(p=0.002, p$ $<0.001$, respectively), and not at TH or L1-4 (Fig. 2b). Intergroup BMD differences over 3 years were significant at FN and TH $(p=0.014, p=$ 0.020 ), but not at L1-4 ( $p=0.250)$ (Fig. 2b). Among the three subgroups (groups I-III), there was a significant negative antibody numberdependent effect on the $\triangle \mathrm{BMD} \%$ at $\mathrm{TH}, 3$ years later ( $p$ for trend 0.021 , Fig. $3 \mathrm{~b}$ ).

\section{Influential factors related to excessive bone loss at three sites}

In univariate logistic analysis, baseline DAS28 $(p=0.016)$, mean DAS28 $(p=0.028), \operatorname{ESR}(p=0.011)$, and BMD at TH $(p=0.017)$ were significantly correlated with excessive bone loss at TH (Table 3). In addition, the presence of seropositivity also showed a significant correlation with excessive bone loss at TH $(p=0.037)$. In multivariate logistic analysis, along with $\mathrm{BMD}$ at $\mathrm{TH}$, the presence of seropositivity increased the potential of excessive bone loss at TH (OR 1.85, 95\% Cl 1.03-3.33, $p=0.039$ ) (Table 3). However, the seropositivity was not significantly associated with excessive bone loss at FN or spine (data not shown).

\section{Table 3}

Predictive values for excessive bone loss $(\triangle B M D \geqq-5 \%)$ at the total hip 


\begin{tabular}{|c|c|c|c|c|}
\hline & $p$ value & OR & $95 \% \mathrm{Cl}$ & $p$ value \\
\hline Age, years & 0.419 & & & \\
\hline Female, n (\%) & 0.206 & & & \\
\hline Postmenopause, n (\%) & 0.922 & & & \\
\hline BMI, kg/m² & 0.754 & & & \\
\hline Comorbidities +, n (\%) & 0.743 & & & \\
\hline \multicolumn{5}{|l|}{ RA-related factors + } \\
\hline Disease duration, years & 0.837 & & & \\
\hline Baseline DAS28-ESR & 0.016 & 1.19 & $0.91-1.55$ & 0.201 \\
\hline Mean DAS28 at follow-up & 0.028 & 1.28 & $0.92-1.78$ & 0.150 \\
\hline HAQ-DI & 0.688 & & & \\
\hline $\mathrm{ESR}, \mathrm{mm} / \mathrm{h}$ & 0.011 & & & \\
\hline \multicolumn{5}{|l|}{ Fracture-related factors ${ }^{b}+$} \\
\hline Parent fractured hip & 0.314 & & & \\
\hline Previous fracture & 0.865 & & & \\
\hline Alcohol & 0.905 & & & \\
\hline Smoking & 0.063 & & & \\
\hline Secondary osteoporosis & 0.332 & & & \\
\hline \multicolumn{5}{|l|}{ Baseline BMD (g/m2) } \\
\hline FN & 0.664 & & & \\
\hline $\mathrm{TH}$ & 0.017 & 21.07 & $3.15-141.03$ & 0.002 \\
\hline L1-4 & 0.278 & & & \\
\hline \multicolumn{5}{|l|}{ FRAX score } \\
\hline Major & 0.793 & & & \\
\hline Hip & 0.714 & & & \\
\hline New incident fracture (3 years), n (\%) & 0.886 & & & \\
\hline Osteoporosis ${ }^{\mathrm{C}}+, \mathrm{n}(\%)$ & 0.767 & & & \\
\hline \multicolumn{5}{|l|}{ Medications } \\
\hline $\mathrm{GC}+, \mathrm{n}(\%)$ & 0.268 & & & \\
\hline $\mathrm{b} / \mathrm{tsDMARDs}^{\mathrm{d}}+, \mathrm{n}(\%)$ & 0.263 & & & \\
\hline $\mathrm{AOT}^{\mathrm{e}}+\mathrm{n}(\%)$ & 0.955 & & & \\
\hline Seropositivity, n (\%) & 0.037 & 1.85 & $1.03-3.33$ & 0.039 \\
\hline Number of antibodies & 0.102 & & & \\
\hline
\end{tabular}

Multivariable analysis was performed after exclusion of ESR due to high correlation.

Abbreviation: BMI, body mass index; DAS28-ESR, disease activity score-28 joint-erythrocyte sedimentation; HAQ-DI: health assessment questionnaire disability index; ESR, erythrocyte sedimentation rate; BMD, bone mineral density; FN, femoral neck; TH: total hip; L1-4, 1st-4th lumbar vertebra; FRAX, fracture risk assessment tool; GC, glucocorticoid; b/tsDMARDs, biologic/target synthetic disease modify antirheumatic drugs, AOT, Anti-osteoporotic therapy; OR, odds ratio; 95\% Cl , 95\% confidence interval

+ , presence

$\triangle \mathrm{BMD}$ means percentage change in BMD (second BMD-baseline BMD/ baseline BMD in percentage) 


\section{Influential factors associated with the number of autoantibodies}

In an attempt to identify the influential factors associated with a greater number of autoantibodies, ordinal logistic regression analysis was performed. After adjusting for the confounding factors (comorbidity, baseline DAS28, osteoporosis, FRAX score (hip), baseline BMD of FN, baseline BMD of TH, GC use), treatment with b/tsDMARDs (OR 1.96, 95\% $\mathrm{Cl} 1.08-3.57, p=0.028)$ and $\triangle \mathrm{BMD} \%$ at TH $(\mathrm{OR} 0.97,95 \% \mathrm{Cl} 0.93$ $0.99, p=0.027$ ) were found to be related to a greater number of autoantibodies.

\section{Discussion}

Our study provides 3-year follow-up results on the impact of seropositivity and the number of autoantibodies on the changes in BMD in patients with RA. We noticed that SPRA patients experienced a significant decrease in BMD at the FN, TH, and L1-4 compared with baseline, but not in SNRA at the TH and L1-4. SPRA patients demonstrated clear bone loss at the TH over time compared to SNRA patients, as indicated by the $\triangle B M D \%$. Furthermore, we recognized that the presence of antibodies had a significant predictive value for $\triangle B M D \geqq-5 \%$ at $T H$, along with baseline BMD at TH. Finally, we found that patients with higher numbers of antibodies had more systemic bone loss at $\mathrm{TH}$.

It has been well documented that RA patients have a higher risk of osteoporosis and fragility fractures than the general population [18]. RAassociated bone loss is not only related to traditional risk factors of osteoporosis (e.g., aging, female sex) but also to factors related to the disease itself (e.g., disease activity and duration, GC use, and functional disability) [20, 21]. However, it is still controversial whether seropositivity affects osteoporosis and BMD changes in RA. Some studies have reported that SNRA patients have a greater chance of developing osteoporosis and lower BMD than SPRA patients [22, 23], but other investigations revealed opposite results [18, 24-26]. These investigations were subjected to a cross-sectional study, which had a small sample size and confounding factors were not adjusted for. In terms of the influential factors screened, we ran binary regression analysis and observed that seropositivity was independently associated with excessive bone loss at TH during the follow-up period. In this investigation, we explored the BMD changes in SPRA and SNRA patients in a 3year, longitudinal, real-world observation study with adequate sample size; we adjusted the analysis, factoring in the confounding factors. The results of our study suggest that seropositivity has a detrimental effect on systemic bone loss in RA patients.

It seems that the greater systemic bone loss observed in SPRA patients is multifactorial. Recent investigations have suggested that not only do SPRA patients have distinct genomic backgrounds [27], clinical presentations [28] and treatment responses [29] from SNRA patients, they also have higher mortality [3]. It raises the possibility that SPRA and SNRA are two distinctive disease entities that mediate the different patterns of systemic bone loss seen in these two subtypes of RA [18]. Current investigations revealed that RA-related factors and its treatment, including baseline DAS28-ESR, ESR, and rate of GC and biologics use, were significantly higher in SPRA than SNRA, which further suggests that these subtypes of RA are different disease entities. The disease activity of RA [20,30] and GC use [21,31] are two of the determinants for RA-related systemic bone loss, suggesting that higher disease activity and more GC usage are the important determinants of greater systemic bone loss and a higher proportion of osteoporosis in SPRA than SNRA in our cohort.

In addition to the indirect effect of different disease patterns on bone loss between SPRA and SNRA, the direct effect of seropositivity on bone loss has been demonstrated. Harre et al. revealed an association between ACPA and serum markers for osteoclast-mediated bone resorption in RA patients [32]. Later on, Krishnamurthy et al. demonstrated that polyclonal ACPAs enhance osteoclast differentiation through a PAD-

dependent IL-8-mediated autocrine loop [33]. A recent study also illustrated that ACPA-positive RA has a higher expression of CXCL2 (which can mediate the osteoclastogenesis in vitro) than ACPA-negative RA [34]. Monocyte lineage challenge with ACPA or RF autoantibodies can induce production of the cytokines TNF-a, IL-1 $\beta$, IL-6, and IL-8 which are directly able to enhance osteoclast formation [35]. All of the evidence suggests that ACPA or RF may induce osteoclastogenesis directly, which might partly mediate the systemic bone loss in RA.

In this study, we also demonstrated that the concurrence of ACPA and RF was linked to more systemic bone loss over time. The additive effect of ACPA and RF on the production of the pro-inflammatory cytokine TNF-a, which is among the most potent cytokines to stimulate osteoclastogenesis, has been noted [10,36,37]. This may explain why there is a 'dose-dependent' effect of antibody number on systemic bone loss (Fig. 2b), and the concomitant presence of ACPA and RF in RA patients had the most detrimental effect on BMD in the three groups. To afford possible predictors of a greater number of autoantibodies, we carried out ordinal regression analysis and found that increased use of b/tsDMARD and more negative $\triangle \mathrm{BMD} \%$ at TH were considered expected factors of the concurrence of RF and anti-ACPA.

Interestingly, we found that not only is substantial bone loss consistent compared with baseline at TH either in SPRA or RA patients with more autoantibodies (Fig. 2a, Fig. 2b), $\triangle$ BMD\% is also more negative (Fig. 3a, Fig. 3b) over time. This is consistent with the findings of previous studies suggesting that RA patients had more bone loss occurred at the hip rather than at the spine [38-40]. Additionally, it is well known that

Page 9/16 
RA predominantly affects the small joints, with early bony erosion in hand joints. Orsolini et al. demonstrated that the independent role of ACPA has a negative titer-dependent effect on baseline BMD at femoral sites, mainly constituted by cortical bone, which is the most interesting in erosion pathogenesis [41]. However, why SPRA patients, especially double seropositive patients, had greater bone loss over time at the hip than the vertebrae remains unknown. We hypothesize, based on the previous studies [38-42] and the findings of this one, that the osteoclastogenesis effect of ACPA and/or RF on cortical and cancellous bones is different. However, its pathogenesis remains to be explored.

The FRAX® was launched in 2008 to allow health providers to estimate individual 10-year probabilities of fragility fractures [43], which is a free, reliable, and validated tool that is used globally. Our previous study demonstrated that RA patients with positive ACPA had a higher 10-year probability of major or hip fractures independent of ACPA levels [14]. Additionally, this study found that there was a significantly higher FRAX score (hip) in SPRA participants regardless of the presence of a single or double-positive antibody (Tables 1 and 2). Despite the higher FRAX score and greater bone loss in SPRA patients, no significant difference was observed in baseline previous fracture and new incident fractures over time between SPRA and SNRA, as well as in groups I-III. This suggests that more RA patients and a longer period of observation are needed to determine whether seropositivity in RA also has a detrimental effect on incident fracture.

The strengths of our study are as follows. As a real-world investigation, we documented as much as possible, the reported clinical variables that may be associated with osteoporosis/fracture in RA patients in order to avoid the possible factors related to BMD changes that were not observed in previous investigations. Aging and sex are two of the most important determinants of BMD. We investigated the impact of ACPA and RF on BMD changes in RA patients by matching age and sex to examine differences between SPRA and SNRA that had not been performed in previous studies. We also explored the additive effect of ACPA and RF on systemic bone loss in RA, which has not been investigated before. Because of the 3-year observation period, we were able to not only investigate the link between the presence of autoantibodies and BMD at baseline, but also to determine the impact of these autoantibodies on BMD changes over time.

This observational study has several limitations. The monocentric observational design used to diagnose RA patients allows us to draw a conclusion from our data about associations, but not about causal relationships. Our patients did not represent an inception cohort; we presented the data at the time of study initiation, not at the time of RA diagnosis. Some of the measurements, including $25(\mathrm{OH})$ vitamin $\mathrm{D}$ and parathyroid hormone, were performed at baseline only (data not shown); serial measurements might be more suitable for exploring the associations. In addition, we did not analyze whether or not seropositivity is an independent factor of systemic bone loss in RA because of the small sample size and short observation period. Finally, we did not check bone markers throughout the study, which hinders the understanding of the pathogenesis of antibodies on systemic bone loss in our cohort.

\section{Conclusion}

Compared to SNRA patients, SPRA patients had a higher prevalence of osteoporosis, consistent bone loss at all sites, and a higher 10-year probability of hip fracture. We also found that the presence of more autoantibodies in RA was associated with more detrimental effects on BMD. We therefore, suggest that more attention should be paid to osteoporosis and systemic bone loss in SPRA patients, especially double antibody-positive patients. As for fragility fractures, further investigations are needed to explore the association between seropositivity in RA and future fractures in the long term.

\section{Abbreviations}

ACPA: anti-citrullinated protein antibodies; ACR: American College of Rheumatology; BMD: bone mineral density; b/tsDMARDs: biological and targeted synthetic disease-modifying antirheumatic drugs; Cl: confidence interval; DAS28-ESR: disease activity score-28 joint-ESR; ESR: erythrocyte sedimentation rate; EULAR: European League Against Rheumatism; FRAX: Fracture Risk Assessment Tool; GC: glucocorticoids; L14; 1st-4th lumbar vertebrae; OR: odds ratio; RA: rheumatoid arthritis; RF: rheumatoid factor; SPRA: seropositive rheumatoid arthritis; SNRA: seronegative rheumatoid arthritis; TH: total hip; FN: femoral neck;

\section{Declarations}

\section{Acknowledgements}

We are indebted to the Special Interest Group of Osteoporosis in the Taiwan Rheumatology Association for guiding the progression of this study. We also appreciate the assistance provided by the Biostatistics Center, Kaohsiung Chang Gung Memorial Hospital. This manuscript was edited by Editage Academic Editing. 


\section{Funding}

The authors disclose receipt of the following financial support for the research, authorship, and/or publication of this article: This work was supported by the grant CMRPG8K0441 from Chang Gung Memorial Hospital (https://www.cgmh.org.tw/), which covered the costs of data collection, input, processing, and publication.

\section{Authors' contributions}

SFY and TTC were responsible for data collection, analysis, manuscript preparation and scientific writing. JFC, YCC, YWW, CYH, HML, and HRH contributed to data collection, manuscript recommendation and editing. All authors reviewed the manuscript. All authors read and approved the final manuscript.

\section{Availability of data and materials}

The datasets generated and analyzed during the current study are not publicly available due to privacy concern of participants but are available from the corresponding author on reasonable request.

\section{Ethics approval and consent to participate}

The Chang Gung Memorial Hospital ethics committee approved the study with approval number 104-3530B and 201901054B0. The study was conducted in accordance with the Declaration of Helsinki. All participants gave their written informed consent to participate.

\section{Consent for publication}

Not Applicable.

\section{Competing interests}

The authors declare that they have no competing interests.

\section{Author details}

${ }^{1}$ Division of Rheumatology, Allergy, and Immunology, Department of Internal Medicine, Kaohsiung Chang Gung Memorial Hospital, Taiwan, R.O.C; ${ }^{2}$ Chang Gung University College of Medicine, Kaohsiung, Taiwan, R.O.C.

\section{References}

1. Smolen JS, Aletaha D, McInnes IB. Rheumatoid arthritis. Lancet. 2016;388:2023-2038.

2. Katchamart W, Koolvisoot A, Aromdee E, Chiowchanwesawakit P, Muengchan C. Associations of rheumatoid factor and anti-citrullinated peptide antibody with disease progression and treatment outcomes in patients with rheumatoid arthritis. Rheumatol Int. 2015;35:16931699.

3. Alemao E, Bao Y, Weinblatt ME, Shadick N. Association of Seropositivity and Mortality in Rheumatoid Arthritis and the Impact of Treatment With Disease-Modifying Antirheumatic Drugs: Results From a Real-World Study. Arthritis Care Res (Hoboken). 2020;72:176-183.

4. Ajeganova S, Huizinga TW. Rheumatoid arthritis: Seronegative and seropositive RA: alike but different? Nat Rev Rheumatol. 2015;11:8-9.

5. Arnett FC, Edworthy SM, Bloch DA, McShane DJ, Fries JF, Cooper NS, et al. The American Rheumatism Association 1987 revised criteria for the classification of rheumatoid arthritis. Arthritis Rheum. 1998;31:315-324.

6. Aletaha D, Neogi T, Silman AJ, Funovits J, Felson DT, Bingham CO 3rd, et al. 2010 Rheumatoid arthritis classification criteria: an American College of Rheumatology/European League Against Rheumatism collaborative initiative. Arthritis Rheum. 2010;62:2569-2581.

7. Gough AK, Lilley J, Eyre S, Holder RL, Emery P. Generalised bone loss in patients with early rheumatoid arthritis. Lancet. 1994;344:23-27.

8. Schett G, Gravallese E. Bone erosion in rheumatoid arthritis: mechanisms, diagnosis and treatment. Nat Rev Rheumatol. 2012;8:656-664.

9. Kocijan R, Harre U, Schett G. ACPA and bone loss in rheumatoid arthritis. Curr Rheumatol Rep. 2013;15:366. 
10. Sokolove J, Johnson DS, Lahey LJ, Wagner CA, Cheng D, Thiele GM, et al. Rheumatoid factor as a potentiator of anti-citrullinated protein antibody-mediated inflammation in rheumatoid arthritis. Arthritis Rheum. 2014;66:813-821.

11. Rantapää-Dahlqvist S, de Jong BA, Berglin E, Hallmans G, Wadell G, Stenlund H, et al. Antibodies against cyclic citrullinated peptide and IgA rheumatoid factor predict the development of rheumatoid arthritis. Arthritis Rheum. 2003;48:2741-2749.

12. Hecht C, Englbrecht M, Rech J, Schmidt S, Araujo E, Engelke K, et al. Additive effect of anti-citrullinated protein antibodies and rheumatoid factor on bone erosions in patients with RA. Ann Rheum Dis. 2015;74:2151-2156.

13. Martin-Mola E, Balsa A, García-Vicuna R, Gómez-Reino J, González-Gay MA, Sanmartí R, et al. Anti-citrullinated peptide antibodies and their value for predicting responses to biologic agents: a review. Rheumatol Int. 2016;36:1043-1063.

14. Cheng TT, Yu SF, Su FM, Chen YC, Su BY, Chiu WC, et al. Anti-CCP-positive patients with RA have a higher 10-year probability of fracture evaluated by FRAX®: a registry study of RA with osteoporosis/fracture. Arthritis Res Ther. 2018;20:16.

15. Kanis JA, Adachi JD, Cooper C, Clark P, Cummings SR, Diaz-Curiel M, et al. Standardising the descriptive epidemiology of osteoporosis: recommendations from the Epidemiology and Quality of Life Working Group of IOF. Osteoporos Int. 2013;24:2763-2764.

16. Cosman F, de Beur SJ, LeBoff MS, Lewiecki EM, Tanner B, Randall S, et al. Clinician's Guide to Prevention and Treatment of Osteoporosis. Osteoporos Int. 2014;25:2359-2381.

17. Lodder MC, Lems WF, Ader HJ, Marthinsen AE, van Coeverden SC, Lips P, et al. Reproducibility of bone mineral density measurement in daily practice. Ann Rheum Dis. 2004;63:285-289.

18. Haugeberg G, Uhlig T, Falch JA, et al. Bone mineral density and frequency of osteoporosis in female patients with rheumatoid arthritis: results from 394 patients in the Oslo County Rheumatoid Arthritis register. Arthritis Rheum. 2000;43:522-530.

19. Genant HK, Wu CY, van Kuijk C, et al. Vertebral fracture assessment using a semiquantitative technique. J Bone Miner Res. 1993;8:11371148.

20. Lodder MC, de Jong Z, Kostense PJ, et al. Bone mineral density in patients with rheumatoid arthritis: relation between disease severity and low bone mineral density. Ann Rheum Dis. 2004;63:1576-1580.

21. Ma CC, Xu SQ, Gong X, et al. Prevalence and risk factors associated with glucocorticoid-induced osteoporosis in Chinese patients with rheumatoid arthritis. Arch Osteoporos. 2017;12:33.

22. Sahatciu-Meka V, Rexhepi S, Manxhuka-Kerliu S, et al. Extra-articular manifestations of seronegative and seropositive rheumatoid arthritis. Bosn J Basic Med Sci. 2010;10:26-31.

23. Arain SR, Riaz A, Nazir L, et al. LOW BONE MINERAL DENSITY AMONG PATIENTS WITH NEWLY DIAGNOSED RHEUMATOID ARTHRITIS. J Ayub Med Coll Abbottabad. 2016;28:175-178.

24. Bruno D, Fedele AL, Tolusso B, Barini A, Petricca L, Di Mario C, Barini A, Mirone L, Ferraccioli G, Alivernini S, Gremese E. Systemic Bone Density at Disease Onset Is Associated With Joint Erosion Progression in Early Naive to Treatment Rheumatoid Arthritis: A Prospective 12Month Follow-Up Open-Label Study. Front Med (Lausanne). 2021;8:613889.

25. Sargın G, Köse R, Şentürk T. Relationship between bone mineral density and anti-citrullinated protein antibody and rheumatoid factor in patients with rheumatoid arthritis. Eur J Rheumatol. 2019;6:29-33.

26. Solomon DH, Finkelstein JS, Shadick N, et al. The relationship between focal erosions and generalized osteoporosis in postmenopausal women with rheumatoid arthritis. Arthritis Rheum. 2009; 60:1624-1631.

27. Padyukov L, Seielstad M, Ong RT, et al. A genome-wide association study suggests contrasting associations in ACPA-positive versus ACPAnegative rheumatoid arthritis. Ann Rheum Dis. 2011;70: 259-265.

28. Gadeholt O, Hausotter K, Eberle H, et al. Differing X-ray patterns in seronegative and seropositive rheumatoid arthritis. Clin Rheumatol. 2019;38:2403-2410.

29. Nordberg LB, Lillegraven $S$, Aga AB, et al. Comparing the disease course of patients with seronegative and seropositive rheumatoid arthritis fulfilling the 2010 ACR/EULAR classification criteria in a treat-to-target setting: 2-year data from the ARCTIC trial. RMD Open. 2018;4: e000752.

30. Hsu CY, Chen JF, Su YJ, et al. Time-averaged disease activity of rheumatoid arthritis associated with long-term bone mineral density changes. Ther Adv Chronic Dis. 2020;11:2040622320981517.

31. Blavnsfeldt AG, de Thurah A, Thomsen MD, et al. The effect of glucocorticoids on bone mineral density in patients with rheumatoid arthritis: A systematic review and meta-analysis of randomized, controlled trials. Bone. 2018;114:172-180.

32. Harre U, Georgess D, Bang H, et al. Induction of osteoclastogenesis and bone loss by human autoantibodies against citrullinated vimentin. J Clin Invest. 2012;122:1791-1802.

33. Krishnamurthy A, Joshua V, Haj Hensvold A, et al. Identification of a novel chemokine-dependent molecular mechanism underlying rheumatoid arthritis-associated autoantibody-mediated bone loss. Ann Rheum Dis. 2016;75:721-729.

Page $12 / 16$ 
34. Wang X, Sun L, He N, et al. Increased expression of CXCL2 in ACPA-positive rheumatoid arthritis and its role in osteoclastogenesis. Clin Exp Immunol. 2021;203:194-208.

35. Steffen U, Schett G, Bozec A. How Autoantibodies Regulate Osteoclast Induced Bone Loss in Rheumatoid Arthritis. Front Immunol. 2019;10:1483.

36. Kitaura H, Zhou P, Kim HJ, et al. M-CSF mediates TNF-induced inflammatory osteolysis. J Clin Invest. 2005;115:3418-3427.

37. Lingampalli N, Sokolove J, Lahey LJ, et al. Combination of anti-citrullinated protein antibodies and rheumatoid factor is associated with increased systemic inflammatory mediators and more rapid progression from preclinical to clinical rheumatoid arthritis. Clin Immunol. 2018;195:119-126

38. Cortet B, Guyot MH, Solau E, et al. Factors influencing bone loss in rheumatoid arthritis: a longitudinal study. Clin Exp Rheumatol. 2000;18:683-690.

39. Haugeberg G, Ørstavik RE, Uhlig T, et al. Bone loss in patients with rheumatoid arthritis: results from a population-based cohort of 366 patients followed up for two years. Arthritis Rheum. 2002;46:1720-1728.

40. Haugeberg G, Helgetveit KB, Førre $\varnothing$, et al. Generalized bone loss in early rheumatoid arthritis patients followed for ten years in the biologic treatment era. BMC Musculoskelet Disord. 2014;15:289.

41. Orsolini G, Caimmi C, Viapiana O, et al. Titer-Dependent Effect of Anti-Citrullinated Protein Antibodies On Systemic Bone Mass in Rheumatoid Arthritis Patients. Calcif Tissue Int. 2017;101:17-23.

42. Bugatti S, Bogliolo L, Vitolo B, et al. Anti-citrullinated protein antibodies and high levels of rheumatoid factor are associated with systemic bone loss in patients with early untreated rheumatoid arthritis. Arthritis Res Ther. 2016;18:226.

43. Kanis JA, Johnell O, Oden A, et al. FRAX and the assessment of fracture probability in men and women from the UK. Osteoporos Int. 2008;19:385-397.

\section{Figures}




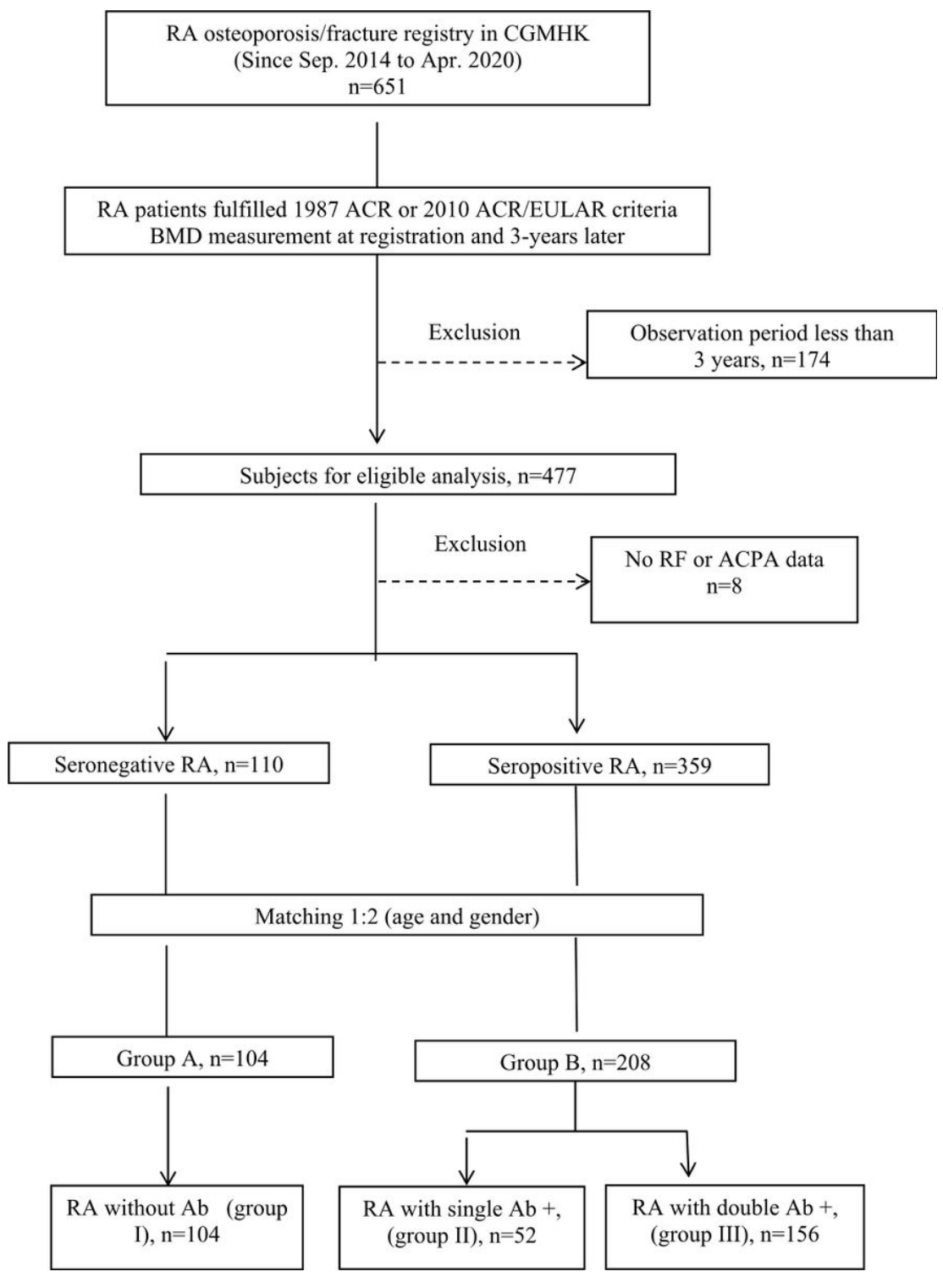

Figure 1

Disposition of participants and grouping. 
$\mathrm{a}$
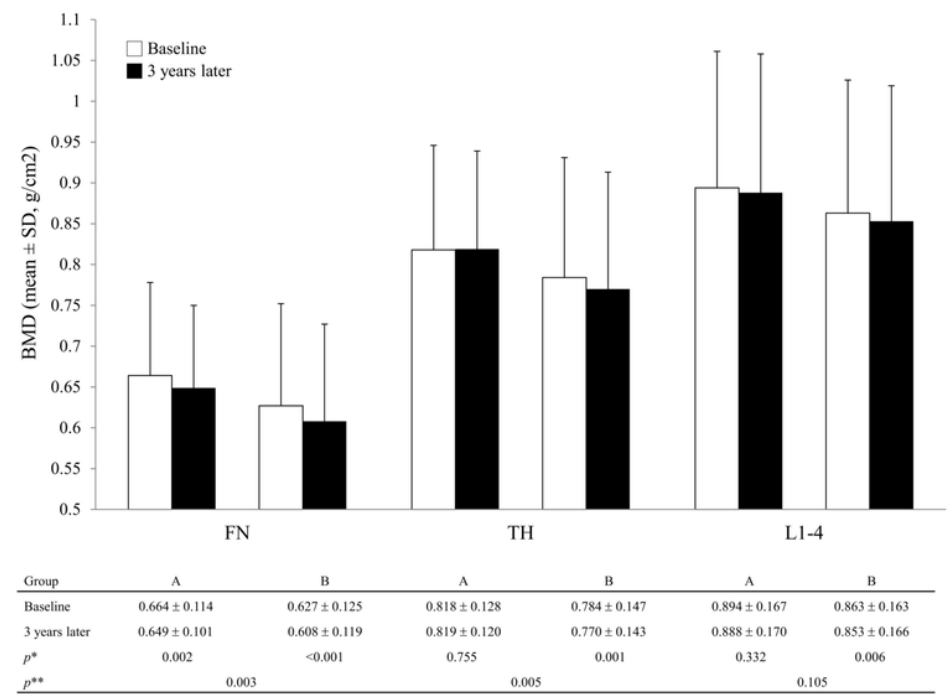

$\mathrm{b}$

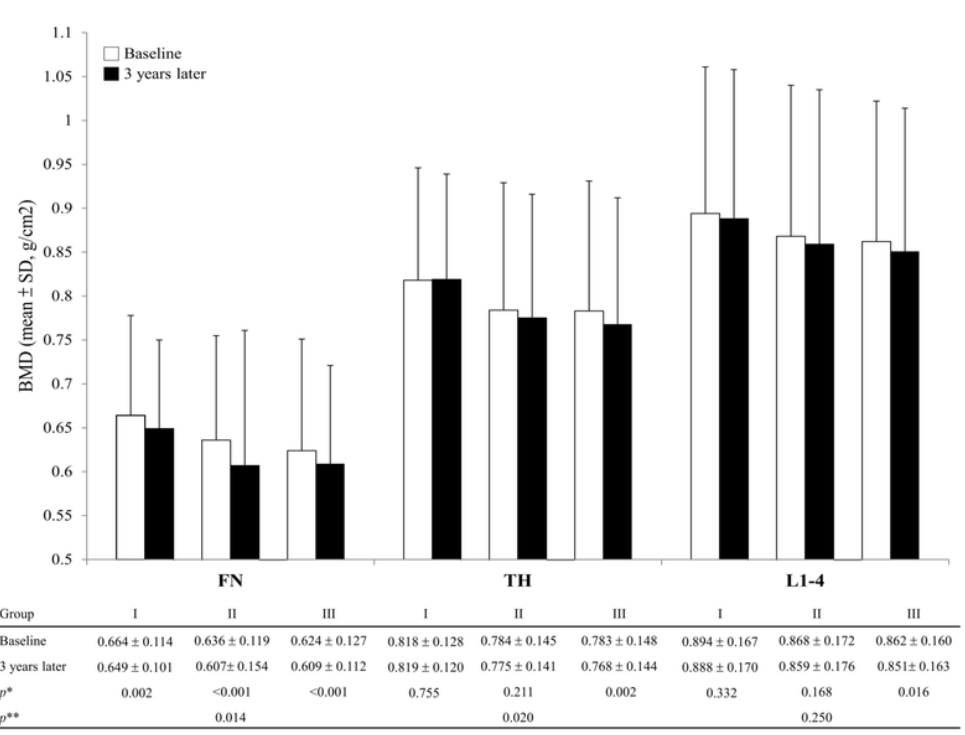

Figure 2

(a) Comparison of BMD between baseline and 3 years later between group A and group B (b) Comparison of BMD between baseline and 3 years later in groups stratified by number of antibody presented, after matching. Statistical method: linear mixed model * in-group comparison ** inter-group comparison. BMD, bone mineral density; FN, femoral neck; L1-4, 1st-4th lumbar vertebra; TH, total hip. 
a

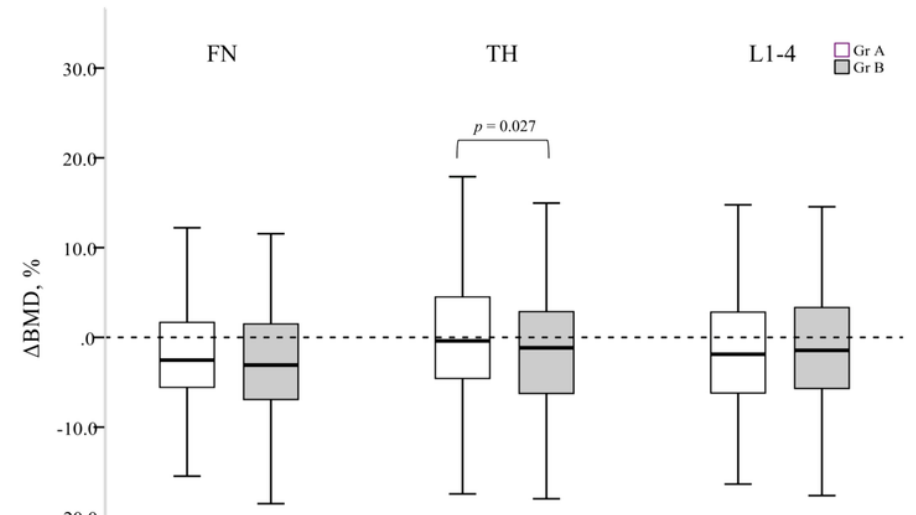

$-20.0$

$-30.0$

$\Delta \mathrm{BMD}, \%(\mathrm{IQR}) \quad-2.5(2.7) \quad-3.1(2.9)$

$-0.3(3.3) \quad-3.1(3.2)$

$-1.5(3.1) \quad-1.5(2.9)$

b

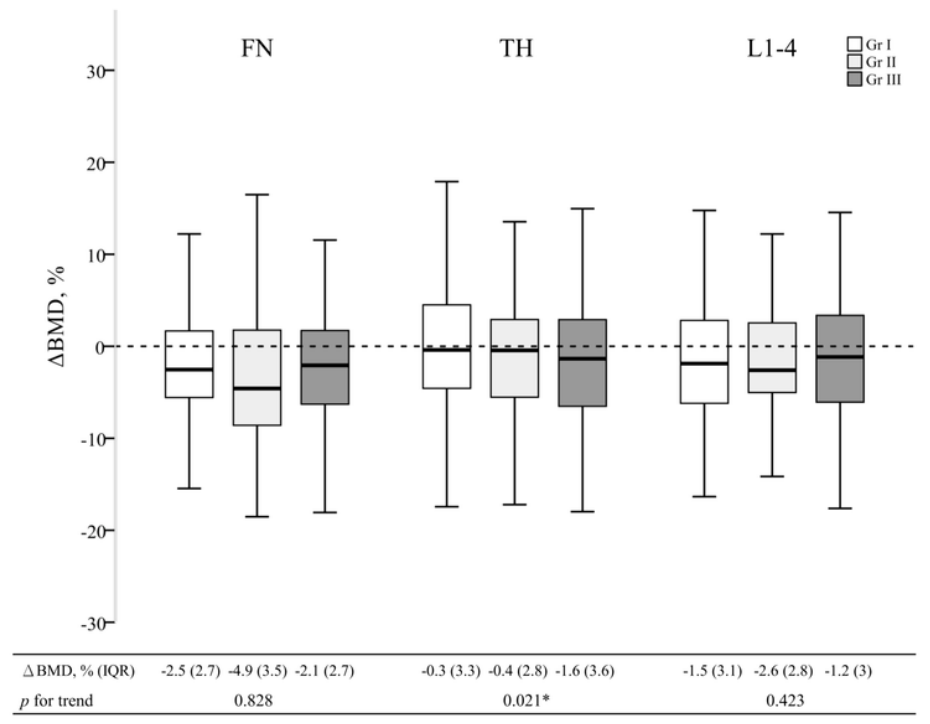

Figure 3

(a) Bone mineral density (BMD) changes from baseline at femoral neck (FN), total hip (TH) and L1-4 between group A and group B patients.

(b) BMD changes from baseline at FN, TH and L1-4 in group I, II and III participants. $\triangle \mathrm{BMD}$ \% (IQR), percentage change in BMD (second BMDbaseline BMD/ baseline BMD in percentage), median\% (interquartile range) 